\title{
Phenomenological study of quarkonium suppression and the impact of the energy gap between singlets and octets
}

\author{
Miguel Ángel Escobedo ${ }^{a, *}$ and Jean-Paul Blaizot ${ }^{b}$ \\ ${ }^{a}$ Instituto Galego de Física de Altas Enerxías (IGFAE), \\ Universidade de Santiago de Compostela, E-15782, Galicia, Spain. \\ ${ }^{b}$ Institut de Physique Théorique, Université Paris Saclay \\ CEA, CNRS, F-91191 Gif-sur-Yvette, France
}

E-mail: miguelangel.escobedo@usc.es, jean-paul.blaizot@ipht.fr

\begin{abstract}
The study of heavy quarkonium suppression in heavy-ion collisions represents an important source of information about the properties of the quark-gluon plasma produced in such collisions. The evolution of the reduced density matrix of heavy quarks inside a quark-gluon plasma is described by a master equation. In a previous work, we found that this master equation needs to take into account the finite energy gap between singlet and octet states in order to lead to the correct thermalization at late times. In this talk, we will discuss the phenomenological consequences of taking into account such energy gap when computing the nuclear modification factor. We will do this in two different scenarios, one using Hard Thermal Loop perturbation theory and another inspired by recent lattice QCD results on the static potential.
\end{abstract}

\footnotetext{
*** Particles and Nuclei International Conference - PANIC2021 ***

*** 5 - 10 September, $2021 * * *$

$* * *$ Online $* * *$
}

${ }^{*}$ Speaker 


\section{Introduction}

Quarkonium suppression is one of the probes of the medium that we can use to characterize the quark-gluon plasma formed in ultrarelativistic heavy-ion collisions. Quarkonium bound states are affected in different ways by the medium. If thermal effects are so strong that they might substantially modify the binding mechanism, then a quantum description is needed. This can be achieved by using the formalism of open quantum systems (for a recent review see [1]). In this formalism the object of study is the reduced density matrix of heavy quarks, whose time evolution is given by a master equation.

Up to now, the master equation of quarkonium has been derived from QCD in the perturbative limit and in the limit $\frac{1}{r} \gg T$ (more details in [1]). There are also many investigations that have used the Abelian approximation. In this work we report on the phenomenological application of the one-gluon exchange model derived in [2]. In [3], it was found that the Langevin equation gives a very good description of quarkonium's evolution in a medium using the Abelian approximation. Next, the QCD case was studied in [4]. Langevin-like equations were found, unfortunately, their range of applicability is much smaller. The reason for this was given in [2]. The energy gap between singlet and octet states must be taken into account in order to obtain a master equation that leads to the correct thermal equilibrium state at large times. In this work, we study what are the phenomenological implications of taking into account this energy gap. More details can be found in [5].

\section{Derivation of the model}

The master equation in the one-gluon exchange approximation, derived in [2], is the following

$$
\begin{aligned}
\frac{\mathrm{d} \mathcal{D}_{Q}}{\mathrm{~d} t}+i\left[H_{Q}, \mathcal{D}_{Q}(t)\right]= & \left.-\int_{\boldsymbol{x} \boldsymbol{x}^{\prime}} \int_{0}^{t-t_{0}} \mathrm{~d} \tau\left[n_{\boldsymbol{x}}^{A}, U_{Q}(\tau) n_{\boldsymbol{x}^{\prime}}^{A} \mathcal{D}_{Q}(t-\tau) U_{Q}^{\dagger}(\tau)\right] \Delta^{>}\left(\tau ; \boldsymbol{x}-\boldsymbol{x}^{\prime}\right)\right) \\
& -\int_{\boldsymbol{x} \boldsymbol{x}^{\prime}} \int_{0}^{t-t_{0}} \mathrm{~d} \tau\left[U_{Q}(\tau) \mathcal{D}_{Q}(t-\tau) n_{\boldsymbol{x}^{\prime}}^{A} U_{Q}^{\dagger}(\tau), n_{\boldsymbol{x}}^{A}\right] \Delta^{<}\left(\tau ; \boldsymbol{x}-\boldsymbol{x}^{\prime}\right),
\end{aligned}
$$

where we have set $t-t^{\prime}=\tau$ and $U_{Q}$ is the $T=0$ time evolution operator. The role of $U_{Q}$ is to inform the master equation of the energy difference between the state at time $t$ and the state at time $t^{\prime}$. Thanks to $U_{Q}$, transitions that release energy are more likely to happen than those that need to absorb it.

It is very challenging to solve eq. (1) in the general case. However, a semi-classical approximation is justified in the limit in which the typical binding energy difference is much larger than the decay width and the master equation is simplified to a rate equation. An argument justifying this can be found in [2]. Using this simplification we can show that the free energy is a monotonically decreasing function in this model, as it should. Since the spectrum of the octet is continuous, we can not apply this rate equation directly. However, since octet to octet transitions are mathematically similar to transitions in an Abelian theory, it is natural to formulate a model consisting in a rate equation for the singlet combined with a Langevin equation for the octet.

In [2], we solved the equations of this model for the case of a static QCD brick. We observed that, indeed, to consider the energy gap reduces significantly the singlet decay width. Finally, we 
observed that the probability that an octet state decays into a singlet is very small for realistic values of the medium volume $\Omega$. Therefore, we can further simplify the equations arriving to the form that we are going to use in our phenomenological application

$$
\frac{d p_{s}}{d t}=-\Gamma_{s} p_{s} .
$$

\section{Phenomenological application}

In this section we will compute $R_{A A}$ assuming a medium that follows a Bjorken evolution. We will do this in two different scenarios. In the first, we consider the case described originally in [2]. A plasma described by Hard Thermal Loop perturbation theory. In the second scenario, we take the real part of the potential from lattice QCD measurements in the static limit [6].

We solve eq. (2) in the following way. We solve the Schrödinger equation with the real part of the potential for several temperatures. From this we obtain the binding energy and the singlet wave-function. We use these quantities as inputs to compute the decay width

$$
\Gamma_{s}=8 \pi \alpha_{s} C_{F} \int_{\mathbf{p}} \mathrm{e}^{-\frac{E_{\mathbf{p}}^{\mathrm{o}}-E^{\mathrm{s}}}{T}} \int_{\mathbf{q}} \Delta^{>}\left(E_{\mathbf{p}}^{\mathrm{o}}-E^{\mathrm{s}}, \mathbf{q}\right)\left|\left\langle\mathrm{s}\left|\sin \left(\frac{\mathbf{q} \cdot \hat{\mathbf{r}}}{2}\right)\right| \mathrm{o}, \mathbf{p}\right\rangle\right|^{2} .
$$

Once we have obtained the decay width for several temperatures, we fit a function to these results. It is this fit function what we use to solve eq. (2). In order to quantify how big is the effect of the energy gap we compare with the results obtained if we use a formula for the decay width that ignores these effects

$$
\Gamma_{s}^{0}=8 \pi \alpha_{s} C_{F} \int_{\mathbf{q}} \Delta^{>}(0, \mathbf{q})\left\langle\mathrm{s}\left|\sin ^{2}\left(\frac{\mathbf{q} \hat{\mathbf{r}}}{2}\right)\right| \mathrm{s}\right\rangle
$$

\subsection{The perturbative case}

The real part of the potential is given by

$$
V_{s}(r)=-\frac{C_{F} \alpha_{s}\left(\mu_{r}\right) e^{-m_{D}\left(T, \mu_{T}\right) r}}{r}
$$

where $\mu_{r}$ and $\mu_{T}$ are (independent) subtraction points. $\mu_{r}$ is either $\frac{1}{2 a_{0}}, \frac{1}{a_{0}}$ or $\frac{2}{a_{0}}$, with $a_{0}$ the Bohr radius. $\mu_{T}$ is either $\pi T, 2 \pi T$ and $4 \pi T$. In this case we found that the condition that the binding energy is much larger than the decay width, needed for the validity of our model, is fulfilled for $\Upsilon(1 S)$ but not for $\Upsilon(2 S)$. The decay width is well fitted by a function of the type $\Gamma \sim a T+b T^{2}$. We observe that the largest source of error is the variation of $\mu_{T}$, changing $\mu_{r}$ having only a mild effect. In this scenario, we let the system evolve until a temperature $T_{f}=200 \mathrm{MeV}$ is reached.

\subsection{The lattice inspired scenario}

In this case, we use the potential

$$
V_{s}(r)=-\frac{\alpha e^{-m_{D} r}}{r}-\sigma r e^{-m_{D} r}\left(1+\frac{2}{m_{D} r}\right) .
$$

This potential was fitted in [6] to static potential data. We observed that the largest source of uncertainty in this scenario comes from the determination of the Debye mass $m_{D}$. In this case, we 

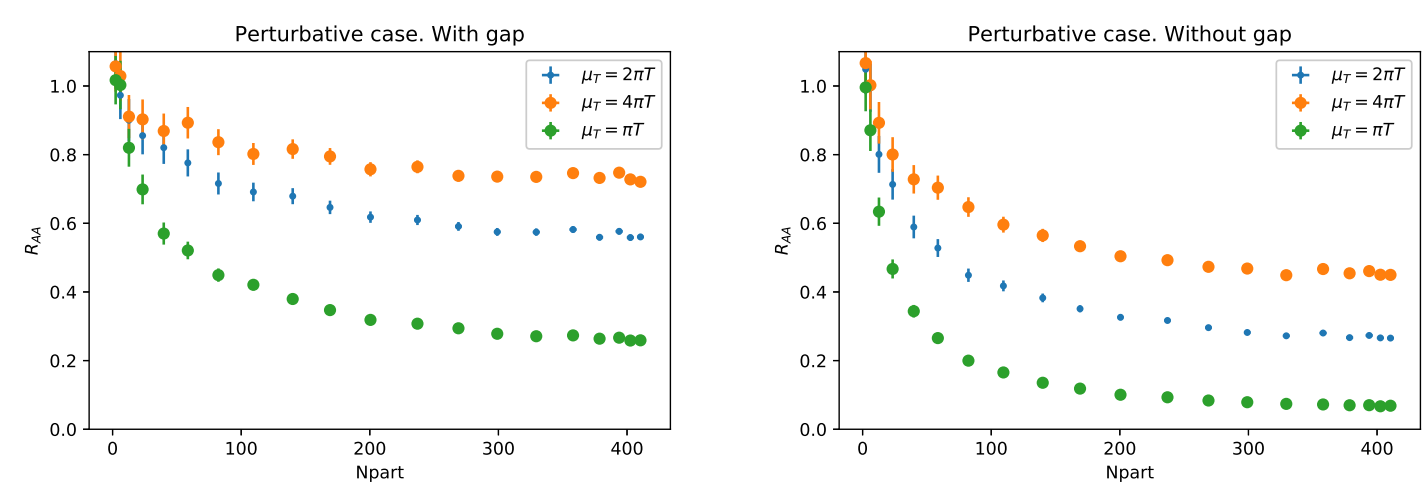

Figure 1: $R_{A A}$ of $\Upsilon(1 S)$ in the perturbative scenario.
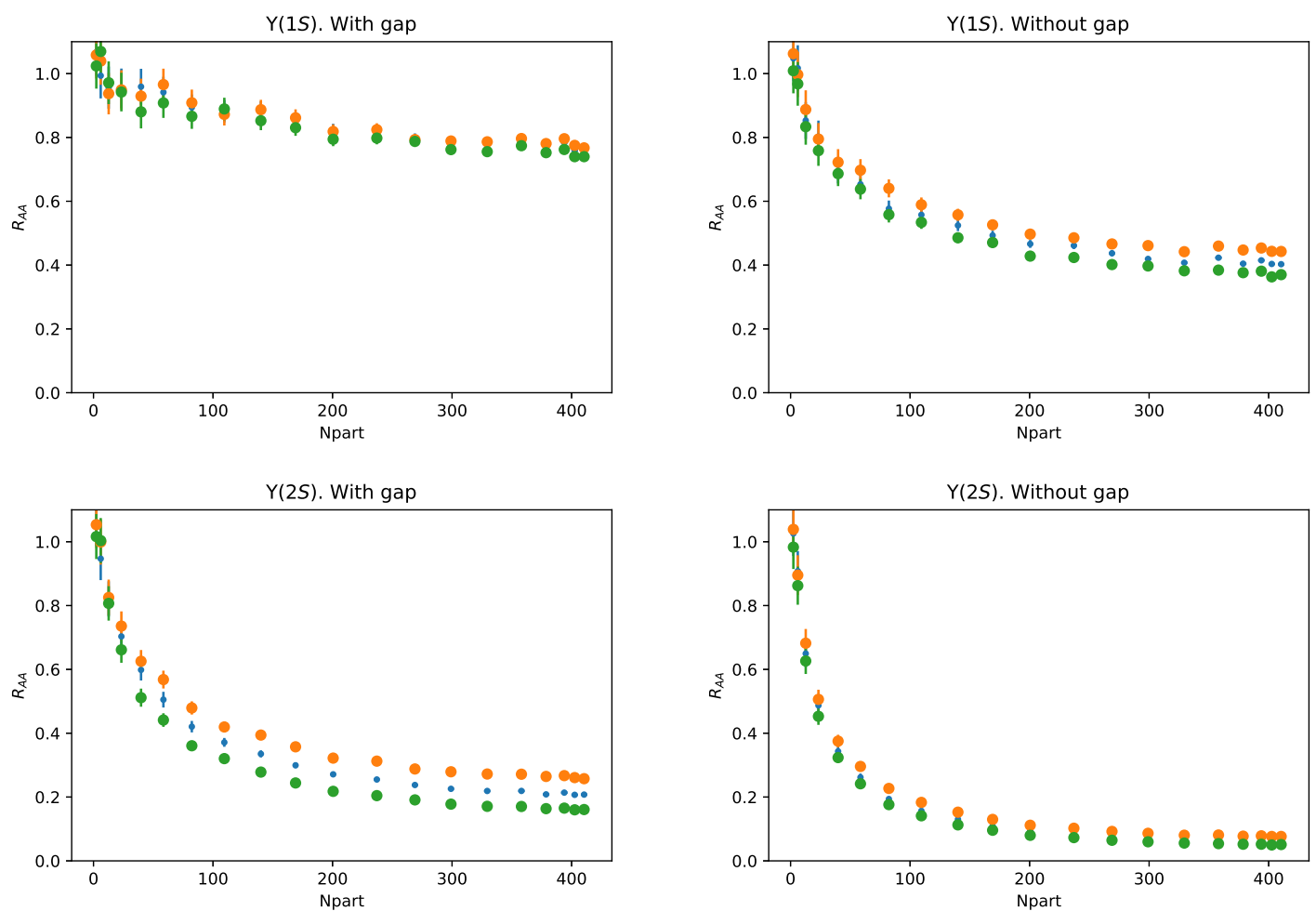

Figure 2: $R_{A A}$ in the lattice inspired scenario.

studied both $\Upsilon(1 S)$ and $\Upsilon(2 S)$. The dependence of the $\Upsilon(1 S)$ decay width with temperature is well fitted by a function $\Gamma(1 S) \sim \tilde{a} T e^{-\tilde{b} / T}$. However, for $\Upsilon(2 S)$ we used the same fitting function that we used for $\Upsilon(1 S)$ in the perturbative case. In both cases we take $T_{f}=180 \mathrm{MeV}$.

\subsection{Results}

We assume that the initial energy density scales like the density of participants and assume that the initial temperature in the center of the most central collisions is $T_{0}(0,0)=475 \times 1.05 \mathrm{MeV}$ for $\sqrt{s}=5.02 \mathrm{TeV}$ collisions at LHC. We assume Bjorken flow with an initial time $t_{0}=0.6 \mathrm{fm}$. With 
this setting, we can compute $R_{A A}$ in the following way

$$
R_{A A}(\boldsymbol{b})=\frac{\int d^{2} \boldsymbol{s} T_{A}(\boldsymbol{s}) T_{A}(\mathbf{s}-\mathbf{b}) S(\boldsymbol{b}, \boldsymbol{s})}{\int d^{2} \boldsymbol{s} T_{A}(\boldsymbol{s}) T_{A}(\mathbf{s}-\mathbf{b})},
$$

where $S(\boldsymbol{b}, \boldsymbol{s})$ is the survival probability. In the perturbative case, the results can be seen in fig. 1 . Regarding the lattice inspired scenario, the results are shown in fig. 2. We expect our model to be more reliable at moderate temperatures (not too central collisions) since then it is more likely that the condition $E \gg \Gamma$ is fulfilled.

\section{Conclusions}

The energy gap between singlets and octets makes the bound state evolution in QCD very different from that of QED. In the limit $E \gg \Gamma$, the master equation can be simplified to a rate equation. Using this model we have computed $R_{A A}$ for $\Upsilon(1 S)$ and $\Upsilon(2 S)$. We see that indeed the energy gap decreases quarkonium suppression almost by a factor of 2 . The effect is larger in the lattice inspired scenario, as we would have expected, since the energy gap is also larger.

\section{Acknowledgments}

M.A.E has received financial support from Xunta de Galicia, by European Union ERDF, by the "María de Maeztu" Units of Excellence program MDM2016-0692, by the Spanish Research State Agency under project FPA2017-83814-P and from the European Research Council project ERC-2018-ADG-835105 YoctoLHC.

\section{References}

[1] Y. Akamatsu, Quarkonium in Quark-Gluon Plasma: Open Quantum System Approaches Re-examined, 2009.10559.

[2] J.-P. Blaizot and M.A. Escobedo, Approach to equilibrium of a quarkonium in a quark-gluon plasma, Phys. Rev. D 98 (2018) 074007 [1803.07996].

[3] J.-P. Blaizot, D. De Boni, P. Faccioli and G. Garberoglio, Heavy quark bound states in a quark-gluon plasma: Dissociation and recombination, Nucl. Phys. A 946 (2016) 49 [1503.03857].

[4] J.-P. Blaizot and M.A. Escobedo, Quantum and classical dynamics of heavy quarks in a quark-gluon plasma, JHEP 06 (2018) 034 [1711. 10812].

[5] J.-P. Blaizot and M.A. Escobedo, Phenomenological study of quarkonium suppression and the impact of the energy gap between singlets and octets, Phys. Rev. D 104 (2021) 054034 [2106.15371].

[6] D. Lafferty and A. Rothkopf, Improved Gauss law model and in-medium heavy quarkonium at finite density and velocity, Phys. Rev. D 101 (2020) 056010 [1906.00035]. 